\title{
Comparison of Bioradiolocation and Respiratory Plethysmography Signals in Time and Frequency Domains on the Base of Cross-Correlation and Spectral Analysis
}

\author{
Maksim Alekhin $\left(\mathbb{D},{ }^{1}\right.$ Lesya Anishchenko ${ }^{\mathbb{D}},{ }^{1}$ Alexander Tataraidze $\mathbb{D}^{1},{ }^{1,2}$ Sergey Ivashov $\mathbb{D}^{1},{ }^{1}$ \\ Vladimir Parashin, ${ }^{2}$ and Alexander Dyachenko $\mathbb{D}^{3,4}$ \\ ${ }^{1}$ Remote Sensing Laboratory, Bauman Moscow State Technical University, 2nd Baumanskaya 5, Moscow 105005, Russia \\ ${ }^{2}$ Department of Biomedical Engineering, Bauman Moscow State Technical University, 2nd Baumanskaya 5, Moscow 105005, Russia \\ ${ }^{3}$ Department of Ecological and Medical Problems, Prokhorov General Physics Institute, Russian Academy of Sciences, Vavilova 38, \\ Moscow 119991, Russia \\ ${ }^{4}$ Laboratory of Cardiorespiratory System Physiology and Biomechanics, Institute of Biomedical Problems, Russian Academy of Sciences, \\ Khoroshevskoye Shosse 76A, Moscow 123007, Russia
}

Correspondence should be addressed to Maksim Alekhin; maksim.alekhin@gmail.com

Received 8 March 2013; Accepted 13 April 2013

Academic Editor: Francesco Soldovieri

Copyright (C) 2013 Maksim Alekhin et al. This is an open access article distributed under the Creative Commons Attribution License, which permits unrestricted use, distribution, and reproduction in any medium, provided the original work is properly cited.

Comparison of bioradiolocation and standard respiratory plethysmography signals during simultaneous registration of different types of the human breathing movements is performed in both time and frequency domains. For all couples of synchronized signals corresponding to bioradiolocation and respiratory plethysmography methods, the cross-correlation and spectral functions are calculated, and estimates of their generalized characteristics are defined. The obtained results consider bioradiolocation to be a reliable remote sensing technique for noncontact monitoring of breathing pattern in medical applications.

\section{Introduction}

Effective noncontact monitoring of breathing pattern is an important aspect of functional diagnostics and vital signs monitoring [1]. In analysis of respiratory movements character, thoracic and abdominal components are usually taken into consideration. This approach is based on the representation of chest wall as a system with two degrees of freedom [2]. Changes in the volume of its thoracic and abdominal components are almost linearly related to the changes of perimeters of correspondent thoracic and abdominal areas of chest wall.

Usually, analysis of respiratory movements and associated changes in volume of chest wall abdominal and thoracic components is carried out applying respiratory plethysmography (RPG) methods [3]. In intensive care units, impedance RPG is widely used. In turn, inductive RPG methods are more popular in polysomnographic laboratories for analyzing respiratory movements during sleep.

Bioradiolocation (BRL) is a modern remote sensing technique allowing to perform noncontact vital signs monitoring of living objects (even behind optically opaque obstacles), on the base of analysis of specific biometric modulation in reflected radiolocation signal [4]. During tidal breathing process, the modulation is mostly determined by reciprocating displacements of skin surface in abdominal and thoracic areas of chest wall due to periodic contractions of respiratory muscles [5]. In medical practice, BRL technology application is known in somnology for noncontact screening of such types of sleep-disordered breathing as sleep apnea and sudden infant death syndromes [6].

Implementation of novel remote respiratory parameter registration techniques in clinical practice requires their mandatory verification with standard contact biomedical 
research methods [3]. The aspect of matching breathing rate (FBR) values registered applying in parallel BRL and RPG methods was previously [7] studied in details. However, analysis of functional relationships between BRL and RPG signals in both time and frequency domains for registration of various respiration types has not been performed yet. The main aim of this study is the comparison of BRL and RPG signals on the basis of cross-correlation and spectral (CCS) analysis of time series [8]. It is necessary for validation of BRL data with standard contact biomedical research technique and to prove reliability and correctness of BRL method application in noncontact remote monitoring of external respiration activity parameters.

\section{Methods of Cross-Correlation and Spectral Analysis}

Generally, for determining functional relationships between the two processes in time and frequency domains, a range of methods is used, based on calculating CCS functions and estimates of their generalized characteristics. The CCS functions used in this study include normalized cross-correlation function (NCCF) and cross-power spectral density function (CPSDF). A couple of uniform time series with synchronized reference points is usually taken as source data for calculating CCS functions [8].

2.1. Normalized Cross-Correlation Function. NCCF describes linear relationship between two signals reflecting similarity of their shape, as well as relative position in time domain. For digital signals $X$ and $Y$, represented by uniform time series with zero mean values, NCCF is calculated as [8]

$$
\rho_{x y}(\tau)=\frac{K_{x y}(\tau)}{\sigma_{x} \cdot \sigma_{y}}=\frac{K_{x y}(\tau)}{\sqrt{K_{x x}(0)} \cdot \sqrt{K_{y y}(0)}}
$$

where $\rho_{x y}(\tau)$-normalized cross-correlation function; $K_{x y}(\tau)$ -cross-correlation function; $K_{x x}(\tau), K_{y y}(\tau)$-auto- correlation functions; $\sigma_{x}, \sigma_{y}$-standard deviations estimates; $f$ frequency; $\tau$-delay time.

The similarity in shape of two signals in time domain is determined by cross-correlation coefficient $\rho_{C}$, equaled to NCCF at zero time delay [8]

$$
\rho_{C}=\rho_{x y}(0)=\frac{K_{x y}(0)}{\sigma_{x} \cdot \sigma_{y}} .
$$

2.2. Cross-Power Spectral Density Function. CPSDF for two signals $X$ and $Y$ is expressed through the NCCF as follows [8]

$$
P_{x y}(f)=4\left[\sum_{\tau=0}^{T} \rho_{x y}(\tau) \cdot e^{-j 2 \pi f t}\right],
$$

where $P_{x y}(f)$-cross-spectral power density function; $\rho_{x y}(\tau)$ -normalized cross-correlation function; $f$-frequency; $T$ the length of the analyzed time period; $\tau$-delay time; $t$ current time.
Knowing the maximum value of $P_{\max }$ for corresponding $f_{o}$, frequency band $B_{e f}=f_{o} \pm \Delta f_{\text {ef }}$ can be determined, in which the main cross-power spectral density of the two processes is concentrated, where $\Delta f_{e f}$ is effective width of crossspectrum in the neighborhood of the extreme frequency $f_{o}$ [8]:

$$
\Delta f_{e f}=\frac{\rho_{x y}(0)}{2 \pi \cdot 4 \cdot P_{\max }}=\frac{\rho_{\kappa}}{8 \pi \cdot P_{x y}\left(f_{o}\right)} .
$$

\section{Experiment}

Three normal male subjects with no respiratory pathologies (age 22-24 years, height $170-176 \mathrm{~cm}$, weight $64-82 \mathrm{~kg}$ ) participated in the experiments for comparing BRL and RPG signals. The studies were carried out on the base of Laboratory of Cardiorespiratory System Physiology and Biomechanics of State Scientific Center of Russian Federation-Institute of Biomedical Problems of Russian Academy of Sciences [9].

3.1. Equipment. BioRascan multifrequency BRL system with a continuous-wave signal and step frequency modulation, developed at Remote Sensing Laboratory of Bauman Moscow State Technical University, was used in the experiments. The unit has 16 operating frequencies in the range from 3.6 to 4.0 GHz. The received signal is filtered by means of an active analog filter with a bandwidth $0.03-5.00 \mathrm{~Hz}$. The sampling rate is $52.1 \mathrm{~Hz}$. Data are recorded in two quadratures. The BRL signal power flux density is $1.36 \mu \mathrm{W} / \mathrm{cm}^{2}$, which grants safety for both patients and medical staff during cardiovascular and respiratory monitoring with BioRascan BRL system application [10].

When comparing BRL and RPG methods during simultaneous data registration, "Respiration-1" instrumental complex (developed by "Biophyspribor" of Federal MedicalBiological Agency of Russian Federation) was applied for RPG recording. Specialized abdominal and thoracic belt monitors with integrated rheostat-based perimetric sensors were used for registering the respiratory movements of the subjects. Signal sampling rate in both cases is $250 \mathrm{~Hz}$. Previously, "Respiration-1" instrumental complex was used in biomedical research program on the Russian Orbital Segment of the International Space Station [11].

3.2. Experimentation. During the set of experiments, a subject was placed on a prepared couch, lying on his back, arms along his sides (Figure 1). Using a tripod, the BioRascan antenna unit was mounted at a distance of $1.0 \mathrm{~m}$ from the subject, directed towards his chest wall area. The thoracic RPG monitor was attached at the middle of the sternum, the abdominal one, at the hypochondrium, passing above the navel. Each experimental recording started with a deep breath to subsequently perform peak-to-peak synchronization of BRL and RPG signals during further stages of CCS analysis.

BRL data was recorded in single-frequency mode with BioRascan bioradar operating on 3.6 GHz. During the set of preliminary experimentation, the visual analysis of BRL signals, registered on each of the 16 frequencies from the range 


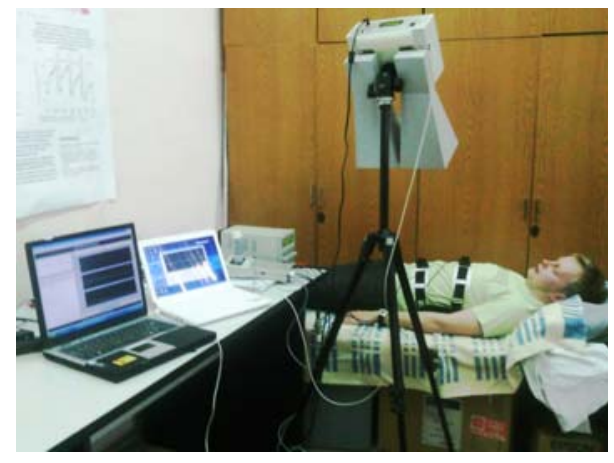

FIGURE 1: Simultaneous registration of BRL and RPG signals during the experiment.

3.6 to $4.0 \mathrm{GHz}$, showed no considerable differences in shape and quality of BRL data. So the selection of another operating frequency from this range has no significant influence on the results of verification of BRL method with RPG in the tasks of noncontact remote monitoring of human breathing pattern. One BioRascan signal quadrature (in phase with the signal of RPG abdominal sensor) on operating frequency of 3.6 GHz was selected for further analysis. This BRL signal generally satisfies the model and experiments described in [1] and is suitable for human breathing pattern monitoring without application of additional demodulation techniques.

An effect of RPG belt perimetric monitors on receiving BRL signal was preliminary estimated. Test signals were recorded: without RPG belt monitors, separately with abdominal and thoracic RPG belt monitors, and with both monitors. No significant changes in the quality of received BRL data were identified in each case according to the results of visual analysis of typical registered BRL signals. Thus, it can be concluded that the presence of RPG belt monitors on the body does not cause significant distortion of the received BRL signal in simultaneous registration of BRL and RPG data.

During the experiment, each of the subjects performed three types of respiratory movements:

(i) A: spontaneous breathing (6 to 15 breaths per minute);

(ii) B: rapid breathing (16 to 30 breaths per minute);

(iii) C: breath holding after a deep breath, followed by continued respiration (Figure 2).

It should be pointed out that correct and effective registration of respiration arrests during sleep for longer than 10 seconds is an important aspect of central sleep apnea screening and is essential in preventive diagnostics of sudden infant death syndrome [12].

In horizontal position (lying on the back), abdominal type of respiration is prevalent. Reciprocating displacements of skin surface in abdominal area mostly contribute to BRL signal during tidal breathing [13]. For this reason, in performing CCS analysis, source data from RPG belt abdominal monitor and BRL signal quadrature on operating frequency of $3.6 \mathrm{GHz}$ were compared.

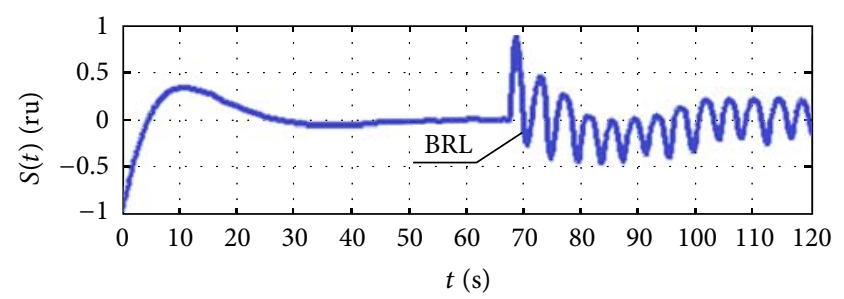

(a)

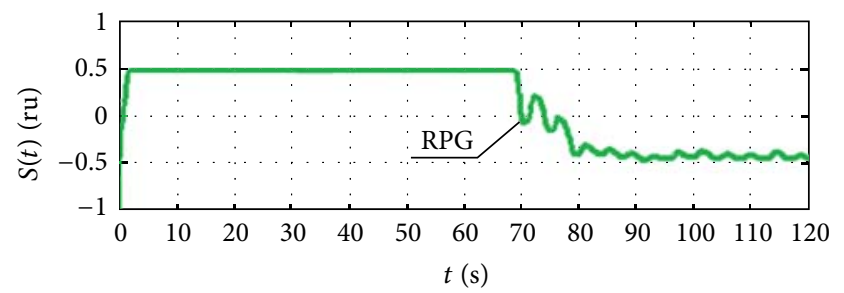

(b)

FIGURE 2: Typical BRL and RPG signals for breath holding episode ((a): BRL signal; (b): RPG signal).

\section{Data Processing}

In the structure of data processing algorithm for CCS analysis of BRL and RPG signals, the following stages can be pointed out:

(i) preprocessing (resampling; smoothing; $Z$-normalization; synchronization of reference points);

(ii) forming two-dimensional arrays of data for couples of $B R L$ and RPG signal realizations;

(iii) construction and analysis of FBR correlation field registered with both BRL and RPG methods;

(iv) calculation and analysis of CCS functions (NCCF; CPSDF);

(v) calculation of estimates of generalized characteristics for CCS functions;

(vi) interpretation and analysis of results.

MATLAB software was used for performing all the data processing routines [14].

4.1. Preprocessing. For each couple of BRL and RPG signals, preprocessing procedure was performed (Figure 3), including the following stages:

(i) resampling of source data using linear interpolation method to set BRL and RPG signals to common sampling rate of $10.0 \mathrm{~Hz}$;

(ii) smoothing the resulting uniform time series using five-point moving average filter to avoid possible high-frequency fluctuations and random bursts of data;

(iii) Z-normalization;

(iv) synchronization of reference points for couples of BRL and RPG signals to form two-dimensional uniform arrays of data for further CCS analysis. 


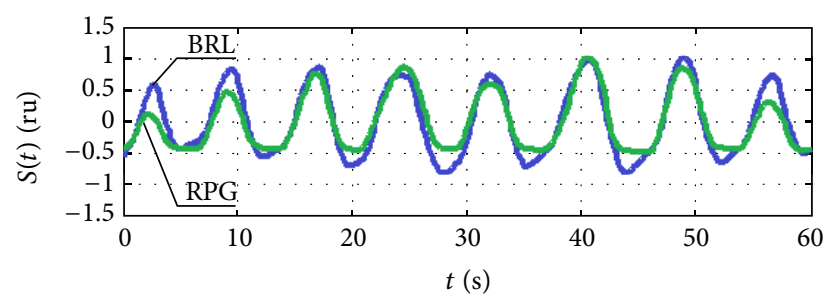

(a)

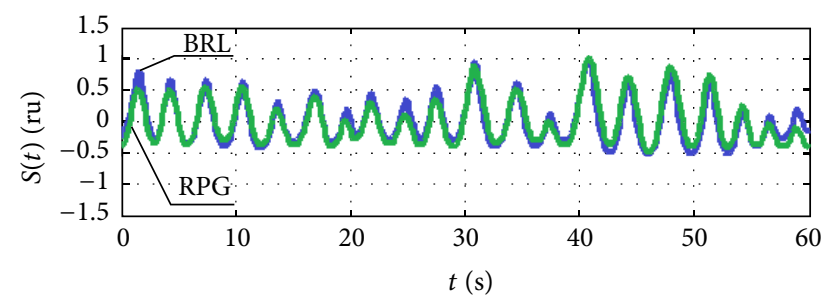

(b)

FIGURE 3: Typical BRL and RPG signals after preprocessing ((a): spontaneous breathing; (b): rapid breathing).

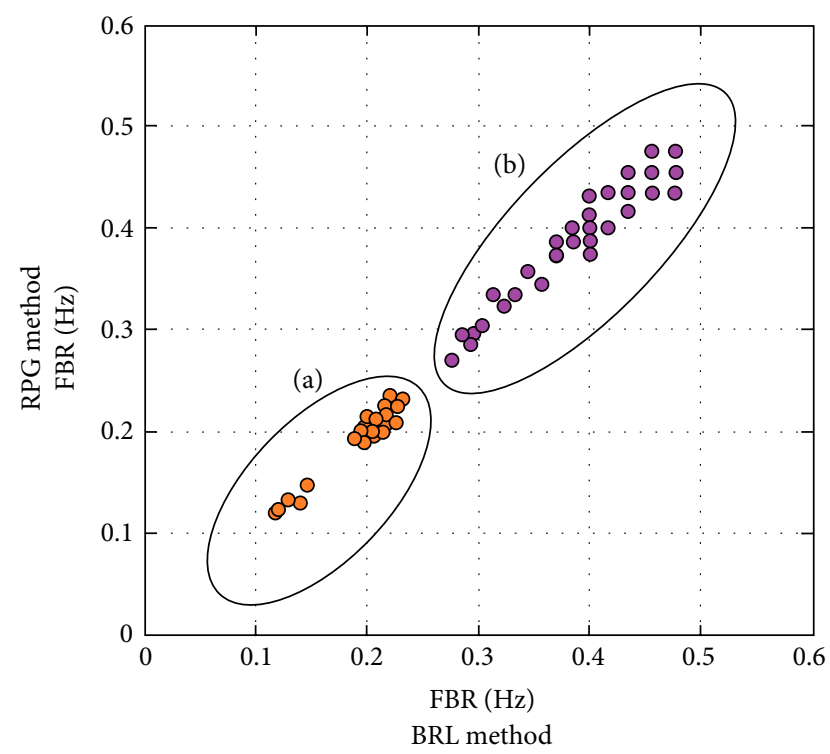

FIgURE 4: Correlation field of FBR calculated for BRL and RPG signals ((a): spontaneous breathing; (b): rapid breathing).

4.2. Analysis of Correlation Field for Breathing Rates. For preliminarily determination of relationship between FBR values obtained from BRL and RPG data, a correlation field was constructed (Figure 4). Each FBR value for BRL and RPG signals realizations was defined as the difference in time between the positions of neighboring peaks, multiplied by the sampling rate $\left(f_{s}=10.0 \mathrm{~Hz}\right)$.

Analysis of the correlation field revealed the strong positive linear relationship between FBR values obtained from BRL and RPG data. Note that it is possible to discriminate spontaneous respiration (typical FBR values in the range $0.12-0.23 \mathrm{~Hz}$ ) and rapid respiration (typical FBR values in the range $0.27-0.48 \mathrm{~Hz}$ ) for all subjects.

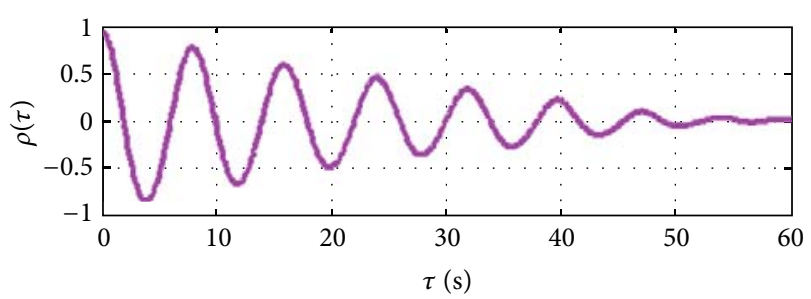

(a)

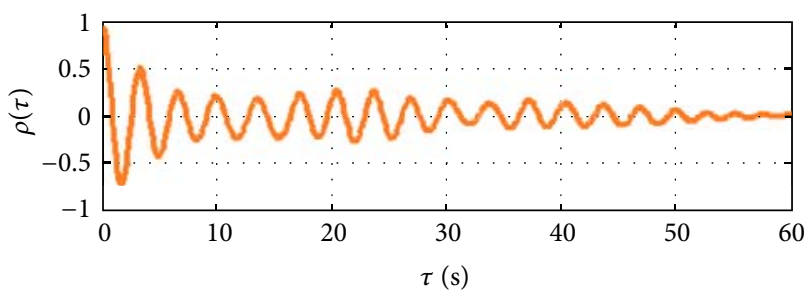

(b)

FIgURE 5: Typical NCCFs for couples of BRL and RPG signals ((a): spontaneous breathing; (b): rapid breathing).

TABLE 1: Generalized characteristics of CCS functions.

\begin{tabular}{lcccc}
\hline Couple of BRL & \multicolumn{5}{c}{ Characteristics of CCS functions } \\
and RPG signals & $\rho_{r b}$ & $f_{o},(\mathrm{~Hz})$ & $P_{r b}\left(f_{o}\right),(\mathrm{ru})$ & $\Delta f_{e},(\mathrm{~Hz})$ \\
\hline $\mathrm{a}$ & & & & \\
& & & & \\
1 & 0.943 & 0.132 & 1.126 & 0.016 \\
2 & 0.922 & 0.22 & 0.784 & 0.023 \\
3 & 0.917 & 0.216 & 1.064 & 0.037 \\
$\mathrm{~b}$ & & & & \\
& & & & \\
1 & 0,935 & 0,312 & 0,379 & 0,049 \\
2 & 0,902 & 0,452 & 0,714 & 0,025 \\
3 & 0,838 & 0,416 & 0,551 & 0,031 \\
\hline
\end{tabular}

1, 2, 3: subject numbers; a: spontaneous breathing; b: rapid breathing.

4.3. Analysis of Normalized Cross-Correlation Function. After forming two-dimensional arrays of uniform mean-corrected time series for couples of BRL and RPG signals realizations, NCCFs were calculated (Figure 5).

As the generalized characteristic of NCCFs, the crosscorrelation coefficients $\rho_{r b}$ were estimated (Table 1), with values ranging from 0.84 to 0.94 that indicate strong linear relationship between BRL and RPG signals in time domain.

4.4. Analysis of Cross-Power Spectral Density Function. Calculations of CPSDF for couples of BRL and RPG signal realizations (Figure 6) were performed using Welch averaged modified periodograms method [15] suppressing the effect of spectrum sidelobes as well as boundary effects and reducing variances of cross-spectral power density estimates.

Among CPSDF generalized characteristics (Table 1), the most informative one is effective cross-spectrum width $\Delta f_{e}$ in the neighborhood of frequency $f_{o}$, corresponding to spectral density maximum. Since the relation $\Delta f_{e} \ll f_{o}$ was satisfied for all the realizations, it can be concluded that CPSDF for BRL and RPG signals is systematically narrowband, and the 


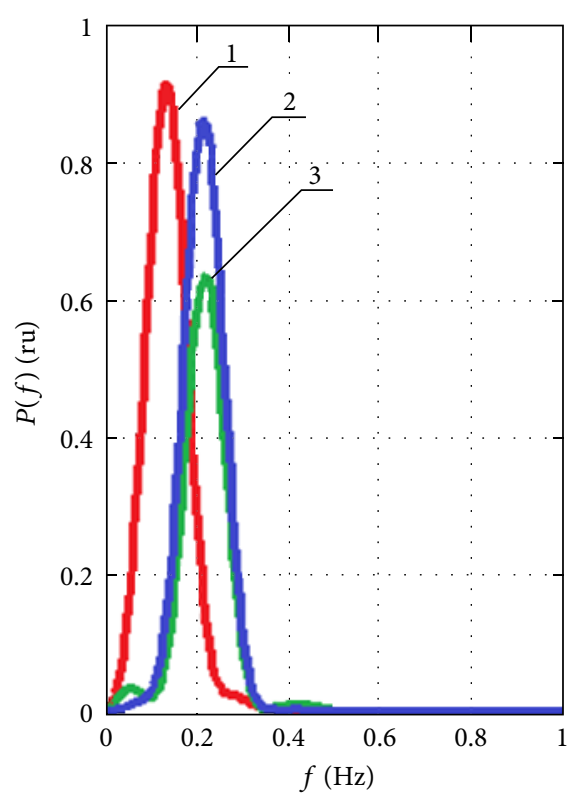

(a)

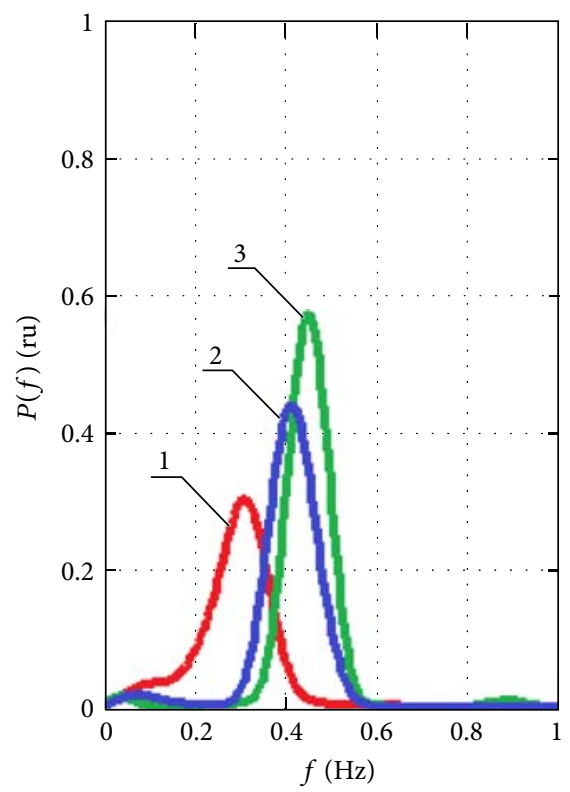

(b)

Figure 6: Typical CPSDFs for couples of BRL and RPG signals (1, 2, 3: subject numbers; (a): spontaneous breathing; (b): rapid breathing).

main cross-power is generally concentrated in the frequency range of the human respiratory movements.

\section{Results}

The estimates of generalized characteristics of CCS functions for analyzed couples of BRL and RPG signals, in accordance with subjects numbers and types of respiratory movements performed in each case, are given in Table 1.
Estimates of the following generalized characteristics of CCS functions for couples of BRL and RPG signals were calculated (1, 2, 3: subject numbers; (a): spontaneous breathing; (b): rapid breathing): $\rho_{C}$ : cross-correlation coefficient; $f_{o}$ : maximum frequency of CSPDF; $P_{r b}\left(f_{o}\right)$ : CPSDF value at the maximum point; $\Delta f_{e}$ : effective width of cross-spectrum (Table 1).

\section{Conclusion}

Verification of BRL with RPG for noncontact remote breathing monitoring in both time and frequency domains was performed on the basis of CCS analysis. The constructed correlation field indicates strong positive linear relationship between FBR values obtained from simultaneously registered BRL and RPG data. The cross-correlation coefficient ranging of 0.84-0.94 indicates strong linear relationship between BRL and RPG signals in time domain. The calculated estimates of cross-spectrum effective width for all the signals realizations reveal the fact that the main cross-power of BRL and RPG signals is concentrated in the narrow frequency range of respiratory movements. Thus, BRL should be considered as a reliable and correct approach for noncontact remote breathing monitoring in biomedical applications.

\section{Acknowledgments}

The research was performed in the framework of the "Active and Passive Microwaves for Security and Subsurface imaging (AMISS)" EU 7th Framework Marie Curie Actions IRSES Project (PIRSES-GA-2010-269157) and supported by the grants of the Ministry of Education and Science of Russian Federation and Russian Foundation for Basic Research.

\section{References}

[1] F. Soldovieri, I. Catapano, L. Crocco, L. Anishchenko, and S. Ivashov, "A feasibility study for life signs monitoring via a continuous-wave radar," International Journal of Antennas and Propagation, vol. 2012, Article ID 420178, 5 pages, 2012.

[2] K. Konno and J. Mead, "Measurement of the separate volume changes of rib cage and abdomen during breathing," Journal of Applied Physiology, vol. 22, no. 3, pp. 407-422, 1967.

[3] "Practice parameters for the indications for polysomnography and related procedures. Polysomnography Task Force, American Sleep Disorders Association Standards of Practice Committee," Sleep, vol. 20, no. 6, pp. 406-422, 1997.

[4] A. S. Bugaev, V. V. Chapursky, S. I. Ivashov, V. V. Razevig, A. P. Sheyko, and I. A. Vasilyev, "Through wall sensing of human breathing and heart beating by monochromatic radar," in Proceedings of the 10th International Conference Ground Penetrating Radar (GPR '04), pp. 291-294, June 2004.

[5] L. Liu, Z. Liu, and B. Barrowes, "Through-wall bio-radiolocation with UWB impulse radar: observation, simulation and signal extraction," IEEE Journal on Selected Topics in Applied Earth Observations and Remote Sensing, vol. 4, no. 4, pp. 791798, 2012.

[6] E. M. Staderini, "UWB radars in medicine," IEEE Aerospace and Electronic Systems Magazine, vol. 17, no. 1, pp. 13-18, 2002. 
[7] J. Muehlsteff, R. Pinter, and G. Morren, "Comparison of respiration rate monitoring with a low-cost Doppler-Radar sensor and inductive thorax-plethysmography," in Proceedings of the World Congress on Medical Physics and Biomedical Engineering (IFMBE '09), pp. 768-771, September 2009.

[8] D. Pollock, Handbook of Time Series Analysis, Signal Processing, and Dynamics, Academic Press, 1999.

[9] Y. Popova, A. Suvorov, A. Dyachenko, and V. Kolesnikov, "Biomechanics and regulation of the external respiration in the conditions of 5-day dry immersion," Aviakosmicheskaia $i$ Ekologicheskaia Meditsina, vol. 45, no. 6, pp. 26-31, 2011.

[10] L. Anishchenko, A. Bugaev, S. Ivashov, and I. Vasilyev, "Application of bio-radiolocation for estimation of the laboratory animals' movement activity," PIERS Online, vol. 5, no. 6, pp. 551554, 2009.

[11] V. Baranov, A. Suvorov, A. Dyachenko, J. Popova, A. Minyaeva, and V. Kolesnikov, "Respiration and respiratory control in longterm spaceflight," in Proceedings of the 17th IAA Humans in Space Symposium, pp. 129-130, 2009.

[12] M. Alekhin, L. Anishchenko, A. Zhuravlev et al., "Estimating diagnostic informativeness of bio-radiolocation pneumography in non-contact screening of sleep apnea syndrome," Biomedical Engineering, vol. 47, no. 2, 2013.

[13] D. A. Korchagina, M. D. Alekhin, and L. N. Anishchenko, "Bioradiolocation method at chest wall motion analysis during tidal breathing," in Proceedings of the 7th European Radar Conference (EuRAD '10), pp. 475-478, October 2010.

[14] D. Higham and N. Higham, MATLAB Guide, SIAM, Philadelphia, Pa, USA, 2005.

[15] P. Welch, "The use of fast Fourier transform for the estimation of power spectra: a method based on time averaging over short, modified periodograms," IEEE Transactions on Audio and Electroacoustics, vol. 15, no. 2, pp. 70-73, 1967. 

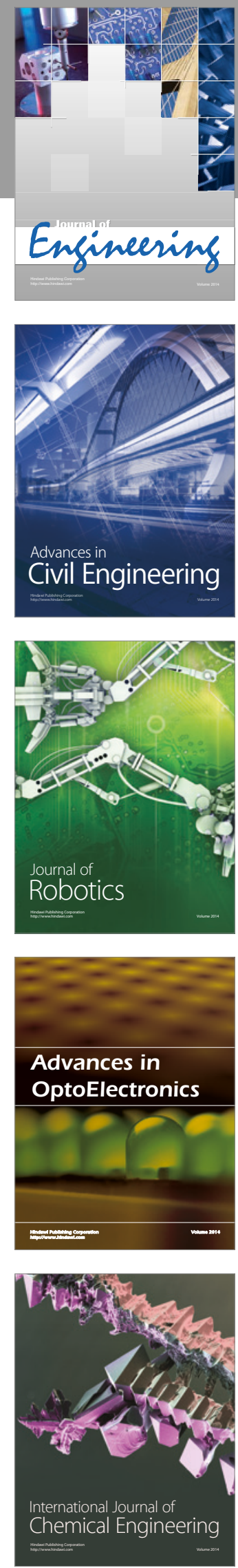

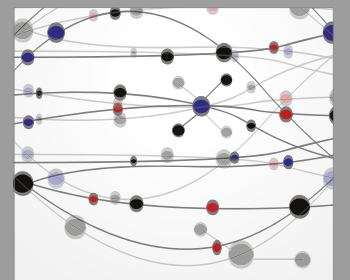

The Scientific World Journal
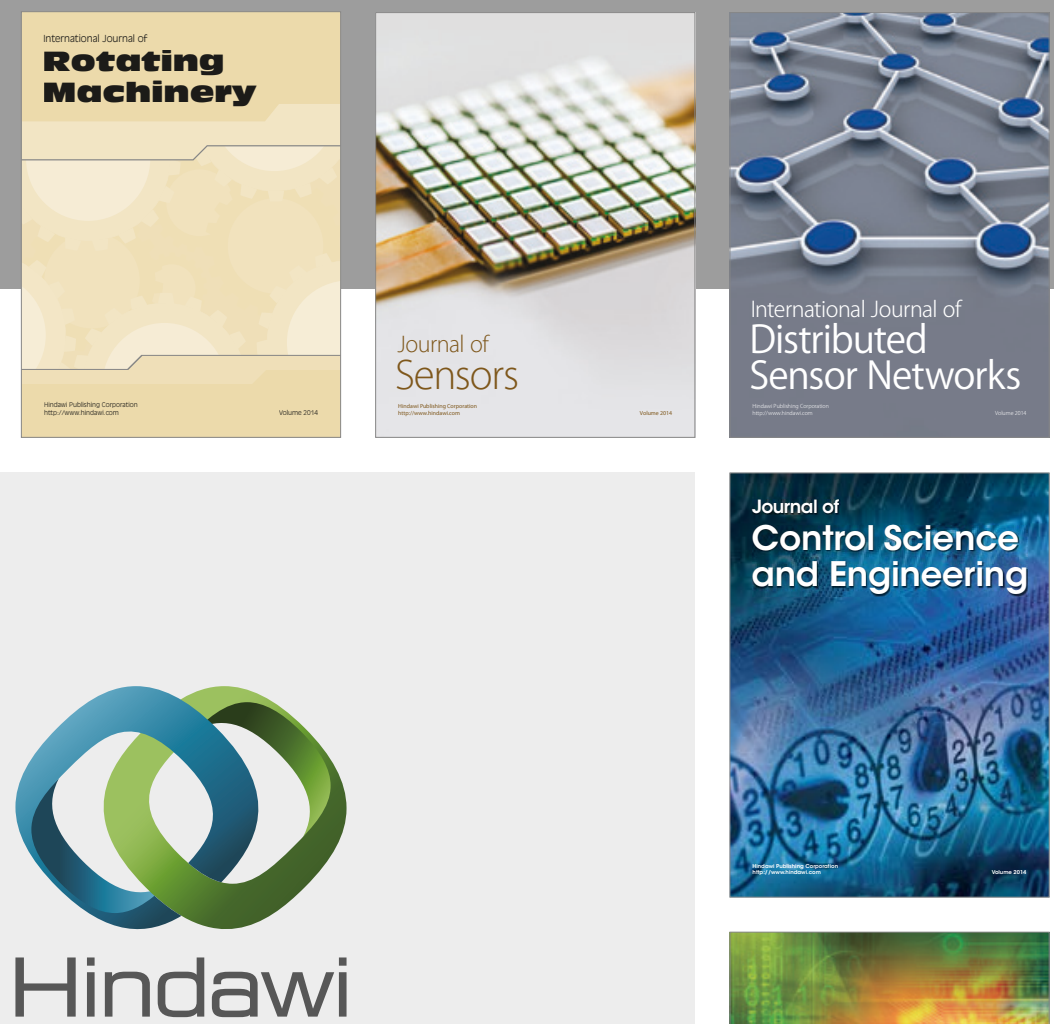

Submit your manuscripts at

http://www.hindawi.com
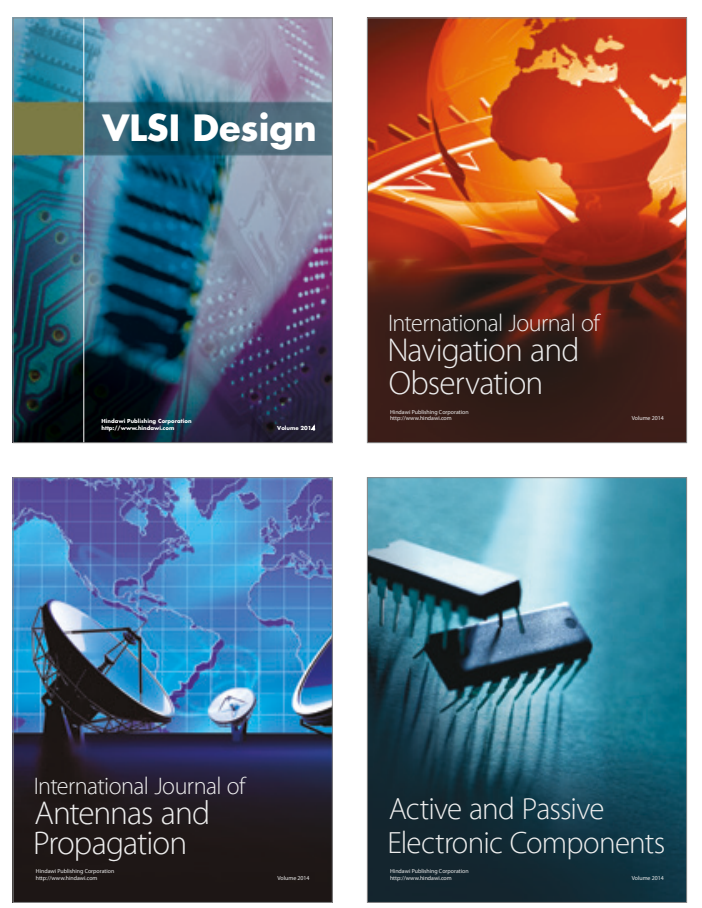
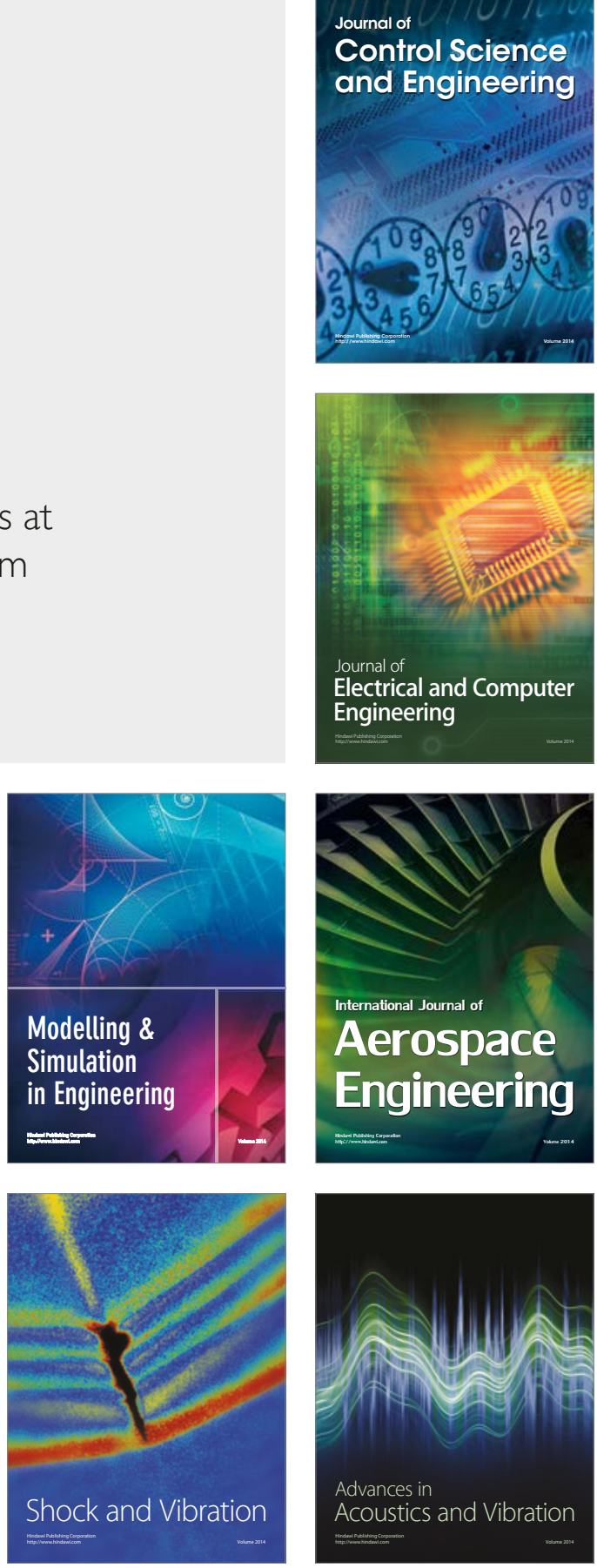